\title{
Clinical significance of evaluating primary lesions in patients with gastric cancer who receive chemotherapy
}

\author{
Kento Nakatani ${ }^{1}$, Wasaburo Koizumi ${ }^{1}$, Katsuhiko Higuchi ${ }^{1}$, Chikatoshi Katada ${ }^{1}$, Toru Sasaki ${ }^{1}$, \\ Norisuke Nakayama ${ }^{2}$, Satoshi Tanabe ${ }^{1}$, and Katsunori Saigenji ${ }^{1}$ \\ ${ }^{1}$ Department of Gastroenterology, Kitasato University School of Medicine, 1-15-1 Kitasato, Sagamihara, Kanagawa 228-8555, Japan \\ ${ }^{2}$ Division of Gastroenterology, Kanagawa Cancer Center, Yokohama, Japan
}

\begin{abstract}
Background. In Western countries, the response of gastric cancer to chemotherapy is evaluated by assessing measurable metastatic lesions (MMLs) according to the response evaluation criteria in solid tumors (RECIST). In Japan, the response of primary lesions is assessed according to local Japanese criteria. We compared the response to chemotherapy as evaluated by these two sets of criteria.

Methods. Patients with unresectable, advanced gastric cancer who had primary lesions and had received first-line chemotherapy were studied. Responses of MMLs were evaluated with RECIST. Responses of primary lesions were evaluated with the Japanese criteria. Median survival times (MSTs) were compared according to treatment response by each set of criteria.

Results. Data from 341 patients were analyzed. Of the 242 patients with MMLs, 108 were MML responders and 134 were MML nonresponders. MST was significantly longer in MML responders (293 days; $95 \%$ confidence interval [CI], 244-342) than in MML nonresponders (159 days; 95\% CI, 127-191; $P<0.0001)$. According to the Japanese criteria, there were 128 primary-lesion responders and 213 primary-lesion nonresponders. MST was significantly longer in responders (304 days; 95\% CI, 266-342) than in nonresponders (168 days; 95\% CI, 143-193, $P<0.0001)$. Of the 99 patients without MMLs, 26 were primary-lesion responders and 73, primary-lesion nonresponders; MST was significantly longer in the former (300 days; 95\% CI, 266-334) than in the latter group (173 days; 95\% CI, 111-235; $P=0.019)$.

Conclusion. The responses of primary lesions according to the Japanese criteria and the responses of MMLs according to the RECIST were both significantly related to the MST. Use of the RECIST alone might bias the evaluation of treatment response because response cannot be evaluated in patients without an MML.
\end{abstract}

Offprint requests to: $\mathrm{K}$. Nakatani

Received: July 7, 2009 / Accepted: December 2, 2009
Key words Gastric cancer · Chemotherapy · Primary lesion · RECIST

\section{Introduction}

The response evaluation criteria in solid tumors (RECIST) [1], reported in 2000, were designed to overcome the problems of the World Health Organization (WHO) criteria. These new criteria permit simpler and standardized evaluations. The three main objectives of the RECIST are to evaluate: (i) tumor response as a prospective endpoint in early clinical trials; (ii) tumor response as a prospective endpoint in more definitive clinical trials designed to provide an estimate of benefit for a specific cohort of patients; and (iii) tumor response as a guide for the clinician and patient or study subjects in making decisions about the continuation of current therapy [1]. The RECIST are designed to evaluate only metastatic lesions and are not relevant to the assessment of primary lesions in gastric cancer.

In Europe and North America, the response of gastric cancer to chemotherapy is thus evaluated by assessing measurable metastatic lesions (MMLs) according to the RECIST; the response of primary lesions is not assessed. In Japan, however, primary lesions have been conventionally evaluated according to the guidelines of the Japanese classification of gastric carcinoma [2]. The main objective of these guidelines is: "... to contribute to improved treatment outcomes in gastric cancer by providing standardized criteria for the assessment and classification of patient information, primary lesions, metastases, therapy, and histopathological findings" [2]. These aims are fundamentally similar to those of the RECIST. Koizumi et al. [3] compared the results of evaluating primary lesions by the Japanese criteria with those obtained by the WHO criteria and reported the advantages and disadvantages of each system. 
The usefulness of the Japanese criteria has also been reviewed by Kiyohashi et al. [4], Yoshida et al. [5], and Ohtsu et al. [6].

In patients with gastric cancer, primary lesions are thought to account for the bulk of the tumor. We therefore doubt whether the value of a drug for the treatment of gastric cancer can be evaluated without assessing the response of primary lesions to such treatment. The aim of this study was to examine the clinical significance of evaluating the response to chemotherapy of primary lesions in gastric cancer as compared with that of evaluating the response of metastatic lesions alone.

\section{Subjects and methods}

\section{Eligibility criteria}

A total of 483 patients with unresectable advanced gastric cancers received first-line chemotherapy at Kitasato University East Hospital from June 1989 through December 2004. Patients who met the following criteria were studied: (i) presence of primary lesions, (ii) age 20 to 80 years, (iii) an Eastern Clinical Oncology Group performance status (PS) of 0 to 2, (iv) adequate organ function, (v) no severe pleural effusion or ascites, and (vi) no other active progressive cancer. The subjects were divided into those with MMLs (measurable group) and those without MMLs (nonmeasurable group) and reviewed retrospectively.

\section{The Japanese classification of gastric carcinoma}

We evaluated primary lesions of gastric cancer according to the Japanese classification of gastric carcinoma. These guidelines evaluate primary lesions on the basis of the results of an upper gastrointestinal X-ray series or endoscopic examination, or both. Lesions are classified as measurable (measurable lesions); able to be evaluated but not measurable (assessable lesions); or diffusely infiltrating, so-called linitis plastica (diffuse lesions). The response of primary lesions to chemotherapy is classified into four categories. Complete response (CR) is defined as the complete disappearance of all lesions for at least 4 consecutive weeks. Partial response (PR) is defined as follows: for measurable lesions, a $50 \%$ or greater decrease in size for lesions that could be measured in two dimensions, or a $30 \%$ or greater decrease in size for lesions that could be measured in only one dimension, maintained for at least 4 consecutive weeks; for assessable lesions, marked shrinkage and flattening of elevated or ulcerated lesions, estimated to be equivalent to a $50 \%$ or greater decrease in size on radiographic or endoscopic examination, maintained for at least 4 consecutive weeks; for diffuse lesions, the gastric lumen of the affected region on radiographic examination shows enlargement of $50 \%$ of greater for at least 4 consecutive weeks. Progressive disease (PD) is defined as an increase in lesion size (of $25 \%$ or greater for measurable lesions), worsening of findings, or the appearance of new lesions. No change (NC) is defined as cases not applicable to any of the above. In the present study, all cases were evaluated by two or more investigators. More than half of the patients were participating in clinical studies; their data were therefore reviewed by an extramural committee.

\section{Clinical characteristics}

The groups were compared with respect to clinical characteristics, including sex ratio, median age, PS, macroscopic type, histopathological type, and treatment regimen. PS was categorized as $0-1$ or 2; macroscopic type as linitis plastica or others, and histopathological type as intestinal type or diffuse type. Treatment regimens were categorized as 5-fluorouracil (5-FU)-based regimens, S-1-based regimens, oral fluorinated pyrimidine-based regimens (excluding those containing S-1), irinotecan-based regimens, or other regimens.

\section{Examination items}

We examined the following six items according to the above-mentioned methods: Exam 1, in the measurable group, survival times were compared between responders $(\mathrm{CR}+\mathrm{PR})$ and nonresponders $(\mathrm{SD}+\mathrm{PD})$ as evaluated by the RECIST alone (i.e., response of MMLs).

Exam 2, in all patients, survival times were compared between responders and nonresponders as evaluated by the Japanese criteria alone (i.e., response of primary lesions).

Exam 3, in the nonmeasurable group, survival times were compared between responders and nonresponders as evaluated by the Japanese criteria alone (i.e., response of primary lesions).

Exam 4, in patients whose MMLs responded to treatment (MML responders) and those whose MMLs did not respond to treatment (MML nonresponders) in the measurable group, survival times were compared between responders and nonresponders as evaluated by the Japanese criteria alone (i.e., response of primary lesions).

Exam 5, in patients whose primary lesions responded to treatment (primary-lesion responders) and those whose primary lesions did not respond to treatment (primary-lesion nonresponders) in the measurable group, survival times were compared between responders and nonresponders as evaluated by the RECIST alone (i.e., response of MMLs). 
Exam 6, the clinical characteristics of the patients were compared between the measurable group and the nonmeasurable group.

\section{Landmark analysis}

The relationships of survival times to the responses of primary lesions and metastatic lesions were estimated using the Kaplan-Meier method in a univariate landmark analysis [7]. The landmark analysis was performed to reduce bias in favor of the responders that was potentially related to time-dependent factors. The landmark for this analysis (day 0) was designated as day 84, which corresponded to the time period required to complete three 28-day courses of chemotherapy (most regimens were 28 days per course). Patients were categorized as responders or nonresponders according to the responses of their primary lesions and metastatic lesions at the landmark time, and differences in their survival times were compared using the log-rank test. Patient characteristics were compared by the $\chi^{2}$ test, Student's $t$-test, and the Mann-Whitney $U$-test. $P$ values of less than 0.05 were considered to indicate statistical significance.

\section{Results}

\section{Characteristics of the patients}

A total of 360 patients met the eligibility requirements. Nineteen patients were excluded from the landmark analysis because of death within 84 days (17 patients) or transfer to other hospitals (2 patients). Data from 341 patients ( 242 patients in the measurable group and 99 in the nonmeasurable group) were included in the land- mark analysis (Fig. 1). Among the total of 341 patients in the landmark analysis, 128 were primary-lesion responders and 213 were primary-lesion nonresponders (Fig. 1). Among the 242 patients in the measurable group, 108 were MML responders and 134 were MML nonresponders (Fig. 1); 102 were primary-lesion responders and 140 were primary-lesion nonresponders. Among the 99 patients in the nonmeasurable group, 26 were primary-lesion responders and 73 were primarylesion nonresponders (Fig. 1).

\section{Exam 1}

On the landmark analysis of the measurable group, the MML responders and MML nonresponders did not differ significantly with respect to patient characteristics such as sex ratio, median age, PS, macroscopic type, histopathological type, or treatment regimen (data not shown). Median survival time (MST) was 293 days (95\% confidence interval [CI], 244-342) in the MML responders and 159 days (95\% CI,127-191) in the MML nonresponders; survival was significantly longer in the responders than in the nonresponders $(P<0.0001$; Fig. 2).

\section{Exams 2 and 3}

There was no significant difference in clinical characteristics between the primary-lesion responders and primary-lesion nonresponders among the total of 341 patients (exam 2, data not shown). The MST of the responders (304 days; 95\% CI, 266-342) was significantly better than that of the nonresponders (168 days; 95\% CI, 143-193; $P<0.0001$; Fig. 3). There was also no significant difference in clinical character-

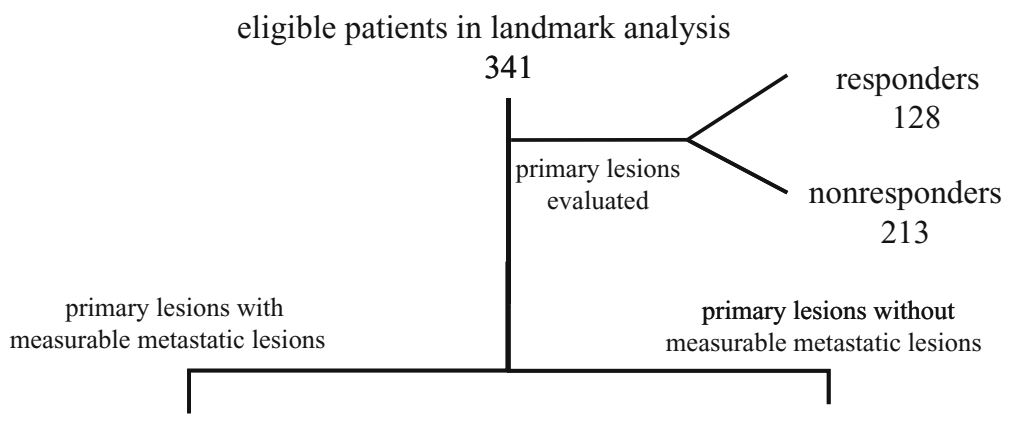

measurable group 242

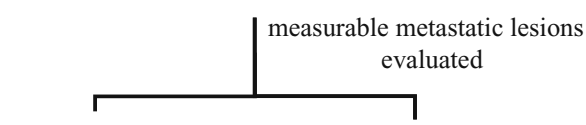

responders 108 nonresponders 134 non-measurable group 99

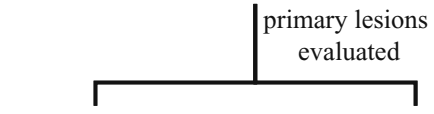

responders 26 nonresponders 73
Fig. 1. Breakdown of patient numbers and categories in the landmark analysis 

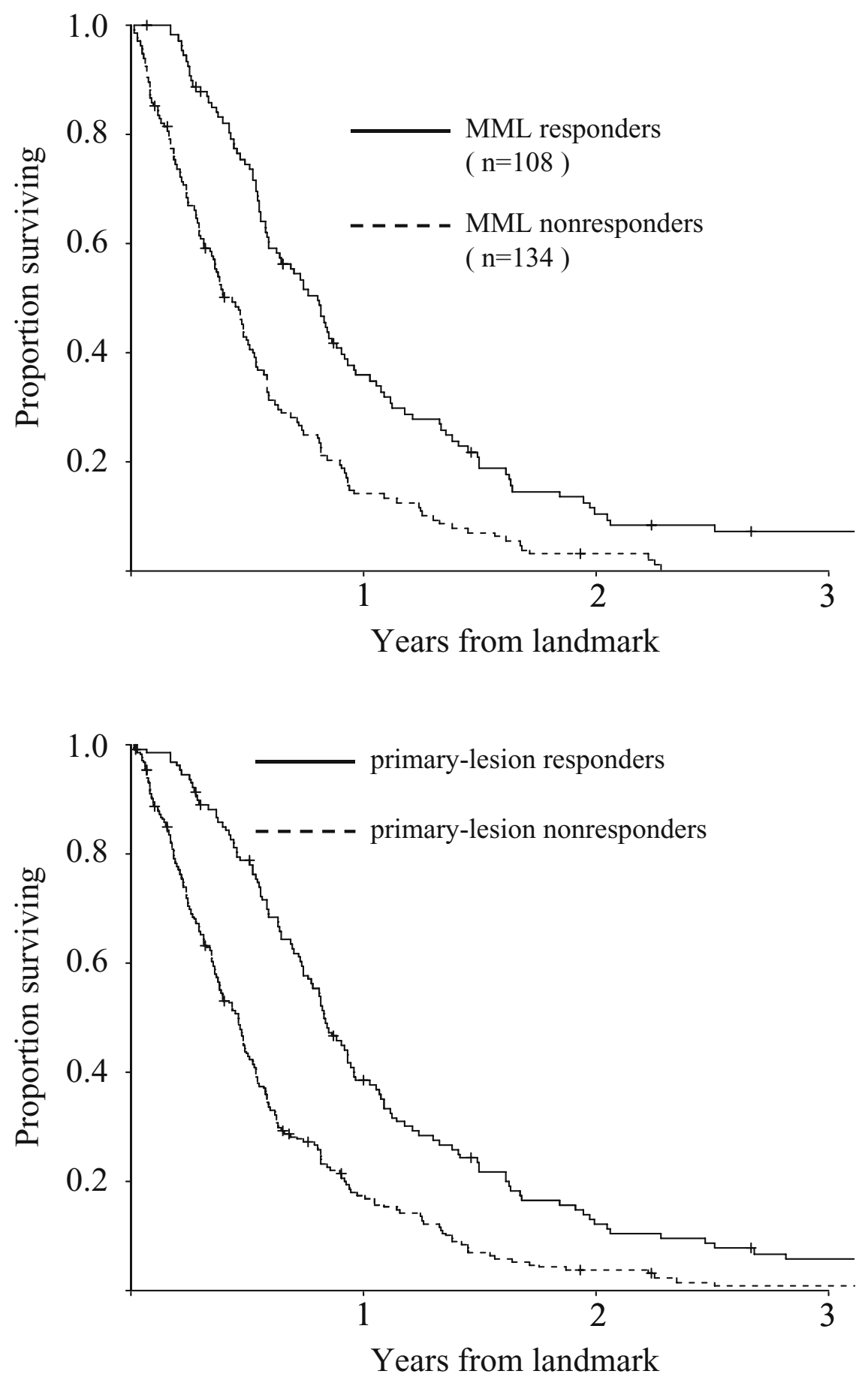

Fig. 2. Comparison of Kaplan-Meier survival curves for the 108 responders and the 134 nonresponders who had measurable metastatic lesions (MMLs) evaluated by the response evaluation criteria in solid tumors (RECIST) in the landmark analysis (log-rank test; exam 1). The median survival time of the MML responders (293 days; 95\% confidence interval [CI], 244-342) was significantly longer than that of the MML nonresponders (159 days; 95\% CI, 127-191; $P<0.0001)$

Fig. 3. Comparison of Kaplan-Meier survival curves for the 128 responders and the 213 nonresponders as evaluated on the basis of primary lesions according to the Japanese criteria in the landmark analysis (log-rank test; exam 2). The median survival time of the primarylesion responders (304 days; 95\% CI, 266-342) was significantly longer than that of the primary-lesion nonresponders (168 days; 95\% CI, 143-193; $P<0.0001$ ) istics between the primary-lesion responders and primary-lesion nonresponders in the nonmeasurable group (exam 3, data not shown). In the nonmeasurable group, MST was 300 days (95\% CI, 266-334) in the primary-lesion responders as compared with 173 days (95\% CI, 111-235) in the primary-lesion nonresponders; survival was thus significantly longer in the responders than in the nonresponders $(P=0.019$, Fig. 4).
Exam 4

The $108 \mathrm{MML}$ responders consisted of 82 primarylesion responders and 26 primary-lesion nonresponders (Table 1). There was no difference in patient characteristics between these groups (data not shown). MST was significantly longer in the responders (310 days; $95 \%$ CI, 275-345) than in the nonresponders (197 days; 95\% CI, 182-212; $P<0.02$; Table 1). The 134 MML nonresponders 


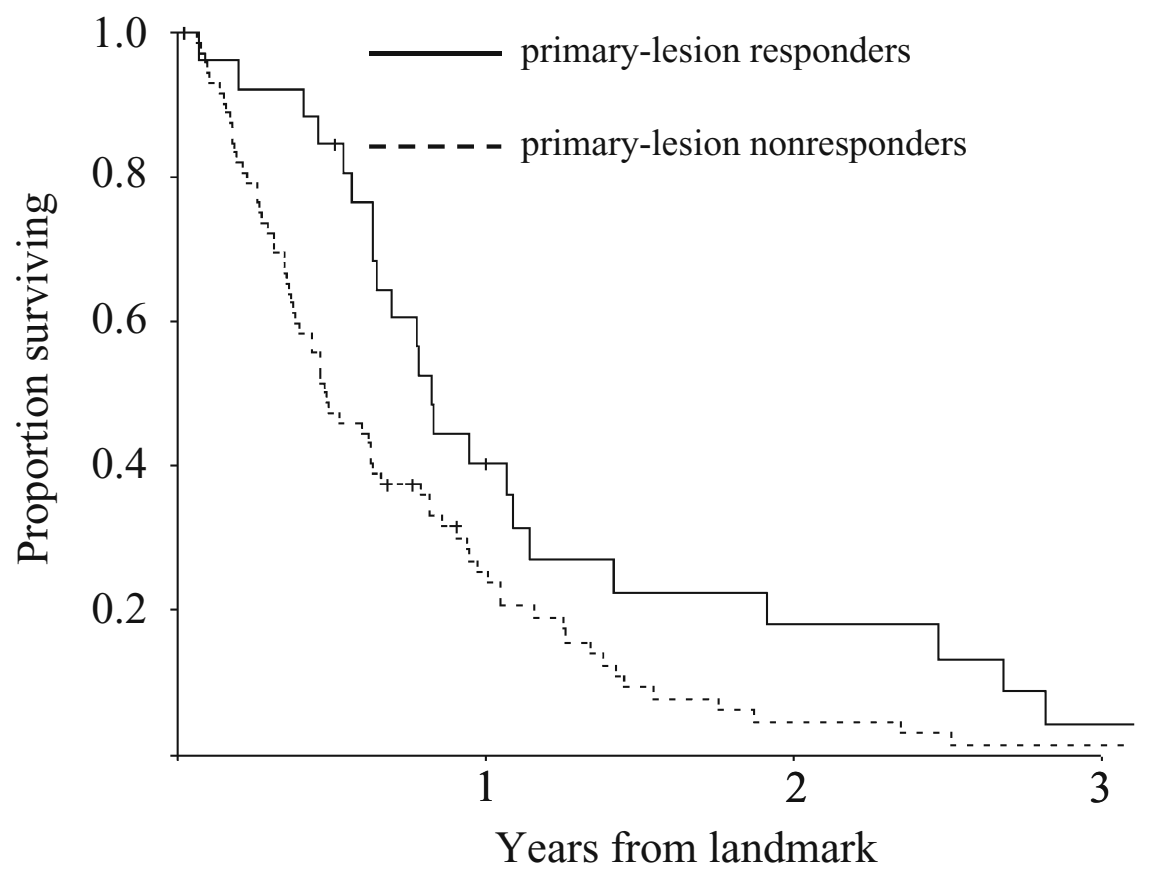

Fig. 4. Comparison of Kaplan-Meier survival curves for the 26 responders and the 73 nonresponders in the nonmeasurable group as evaluated on the basis of primary lesions according to the Japanese criteria in the landmark analysis (log-rank test; exam 3). The median survival time of the primary-lesion responders (300 days; 95\% CI, 266-334) was significantly longer than that of the primary-lesion nonresponders (173 days; 95\% CI, 111-235; $P=0.019)$

Table 1. Comparison of survival times according to treatment response for the 242 patients in the measurable group in the landmark analysis

\begin{tabular}{lcc}
\hline & $\begin{array}{c}\text { Primary-lesion responders }(n=102) \\
\text { MST; days }\end{array}$ & $\begin{array}{c}\text { Primary-lesion nonresponders }(n=140) \\
\text { MST; days }\end{array}$ \\
\hline MML responders $(n=108)$ & $310[275-345](n=82)$ & $197[182-212](n=26)$ \\
MML nonresponders $(n=134)$ & $269[199-339](n=20)$ & $140[107-173](n=114)$ \\
$P$ value & NS & NS
\end{tabular}

In the measurable metastatic lesion (MML) responders and the MML nonresponders, responses of the primary lesions were significantly related to median survival time (MST) (exam 4). In the primary-lesion responders and the primary-lesion nonresponders, however, the responses of MMLs were not related to median survival time (exam 5). These findings indicated that the response of the primary lesions was more strongly related to median survival time than was the response of the metastatic lesions. The median survival time is indicated in days, with $95 \%$ confidence intervals (95\% CI, days) in brackets. The numbers of patients are shown in parentheses. $P$ values of less than 0.05 were considered to indicate statistical significance

NS, not significant

consisted of 20 primary-lesion responders and 114 primary-lesion nonresponders (Table 1). There was no difference in patient characteristics between these groups (data not shown). MST was significantly longer in the responders (269 days; $95 \%$ CI, 199-339) than in the nonresponders (140 days; $95 \%$ CI, $107-173 ; P<0.01$; Table 1).

\section{Exam 5}

No significant differences in MST were found between the $82 \mathrm{MML}$ responders and the $20 \mathrm{MML}$ nonresponders among the 102 primary-lesion responders or between the 26 MML responders and 114 MML nonresponders among the 140 primary-lesion nonresponders (Table 1).

\section{Exam 6}

When clinical characteristics were compared between the 242 patients in the measurable group and the 99 patients in the nonmeasurable group (Table 2), the groups were found to differ significantly with respect to macroscopic type, histopathological type, and treatment regimen. The proportions of patients with linitis plastica and undifferentiated types of gastric cancer were significantly greater in the nonmeasurable group.

\section{Discussion}

The RECIST are used worldwide for evaluation of the response of solid tumors to chemotherapy. The validity 
Table 2. Comparison of demographic characteristics between the measurable group and the nonmeasurable group

\begin{tabular}{|c|c|c|c|}
\hline & Measurable group; total (\%) & Nonmeasurable group; total (\%) & $P$ value \\
\hline Number of patients & 242 & 99 & \\
\hline Median age; years (range) & $63(26-80)$ & $61(28-79)$ & NS \\
\hline \multicolumn{4}{|l|}{ Sex ratio } \\
\hline Male & $168(69.4)$ & $61(61.6)$ & \multirow[t]{2}{*}{ NS } \\
\hline Female & $74(30.6)$ & $38(38.4)$ & \\
\hline \multicolumn{4}{|l|}{ Performance status } \\
\hline $0-1$ & $218(90.1)$ & $85(85.9)$ & \multirow[t]{2}{*}{ NS } \\
\hline 2 & $24(9.9)$ & $14(14.1)$ & \\
\hline \multicolumn{4}{|l|}{ Macroscopic type } \\
\hline Linitis plastica & 45 (18.6) & $54(54.5)$ & \multirow[t]{2}{*}{$<0.000001$} \\
\hline Others & $197(81.4)$ & $45(45.5)$ & \\
\hline \multicolumn{4}{|l|}{ Histopathological type } \\
\hline Intestinal & $87(36.0)$ & $16(16.2)$ & \multirow[t]{2}{*}{$<0.001$} \\
\hline Diffuse & $155(64.0)$ & $83(83.8)$ & \\
\hline \multicolumn{4}{|l|}{ Treatment regimen } \\
\hline 5-FU-based & $72(29.8)$ & $53(53.5)$ & \multirow[t]{5}{*}{$<0.001$} \\
\hline S-1-based & $96(39.6)$ & $26(26.3)$ & \\
\hline Oral fluorinated pyrimidine-based & $56(23.1)$ & $14(14.2)$ & \\
\hline Irinotecan-based & $12(5.0)$ & $2(2.0)$ & \\
\hline Others & $6(2.5)$ & $4(4.0)$ & \\
\hline
\end{tabular}

The macroscopic type, histopathological type, and treatment regimen differed significantly between the groups (exam 6) 5-FU, 5-fluorouracil

of these criteria is beyond dispute. In exam 1 (Fig. 2) in the present study, the response of metastatic lesions as evaluated by the RECIST sufficiently reflected outcomes. This finding suggested that it is unnecessary to evaluate primary lesions of gastric cancer, supporting the validity of the RECIST. However, how to assess the many cases that cannot be evaluated by the RECIST remains an important problem.

A total of $99(29.0 \%)$ of the 341 patients in the present study lacked MMLs; response therefore could not be evaluated by the RECIST (Fig. 1). Thus, nearly $30 \%$ of the patients would have been excluded from the study if responses had been assessed using only the RECIST. The enrolment of the target number of patients is critical to the success of clinical studies. Increased numbers of ineligible patients may prolong the study period. In our study, however, primary lesions could be evaluated by the Japanese criteria in all patients with nonmeasurable metastatic lesions. Moreover, exams 2 and 3 indicated that the response of the primary lesions was correlated with outcomes in patients with gastric cancer (Figs. 3 and 4). These results suggested that the evaluation of primary lesions according to the Japanese criteria was a valid method for the assessment of response, confirming the results of previous studies [3-6]. Our results suggest that the response of primary lesions to chemotherapy should be evaluated if possible in studies of gastric cancer. The Japanese criteria can contribute to this goal because they are designed to evaluate primary lesions.

The results of exams 4 and 5, which evaluated survival times in specific subgroups of patients, indicated that the response of the primary lesions might be more closely related to outcomes than the response of the metastatic lesions (Table 1 ). These results suggest that only primary lesions should be evaluated. Moreover, the results of exam 2, including all patients, showed that it was sufficient to evaluate only primary lesions, because their response was closely linked to outcomes (Fig. 3). Theoretically, outcomes should be better in patients whose primary lesions respond to treatment, because such lesions account for the bulk of tumor in most cases of gastric cancer. Because the RECIST are a simplified, standardized version of the WHO criteria, endoscopic and radiographic examinations are not used for evaluation, for the sake of convenience. Most gastrointestinal oncologists in Japan can correctly interpret diagnostic images and quantitatively evaluate findings on upper gastrointestinal radiographic and endoscopic examinations. In Western countries, however, these assessments are often difficult to perform because of logistic factors and cost restraints.

In the present study, the results of exam 6 indicated that the prevalences of linitis plastica and undifferentiated types of cancer may be lower in studies of patients whose lesions can be evaluated with the RECIST than in the general population of patients with gastric cancer (Table 2). Maruyama et al. [8] have reported that the linitis plastica form of gastric cancer has particularly poor outcomes, requiring caution in evaluation and treatment. Sensitivity to anticancer agents has been reported to depend on the histopathological type of cancer. Undifferentiated adenocarcinoma responds well to alternating chemotherapy with methotrexate and 
5-fluorouracil [9]. Konishi et al. [9] reported that all 9 of the 38 patients $(23.7 \%)$ with gastric cancer who responded to alternating chemotherapy with methotrexate and 5-fluorouracil had undifferentiated adenocarcinoma (diffuse type), whereas none of the patients with well-differentiated adenocarcinoma (intestinal type) responded to treatment. Agents that are particularly effective against the linitis plastica form of gastric cancer may thus produce a poor response when evaluated according to the RECIST, delaying the development of chemotherapy for this type of gastric cancer. We believe that the treatment response of the linitis plastica form of gastric cancer must be evaluated on the basis of the primary lesions, as recommended by the guidelines of the Japanese classification of gastric carcinoma. An inherent risk of using the RECIST for the evaluation of treatment response in gastric cancer is the potential introduction of bias with respect to the demographic characteristics of the subjects, because patients without MMLs are excluded from analysis.

Our present results indicate that, for determining response to chemotherapy in patients with gastric cancer, the primary lesions should be evaluated. Until the time that computed tomography (CT) scanning can accurately assess tumors in hollow organs, upper gastrointestinal radiography and endoscopy remain essential examinations for the evaluation of response. Recent advances in CT scanning have led to the development of multidetector row CT and three-dimensional CT. Several studies have reported that these diagnostic techniques are useful for quantitatively evaluating primary cancers of the intestine [10-12]. However, such techniques are not yet generally available; future developments are awaited.

In conclusion, our results showed that the evaluation of treatment response according to the RECIST was suitable for patients with MMLs in studies of gastric cancer. By using the Japanese criteria, however, the response of primary lesions could be evaluated in all patients without MML and correlated with outcomes. The responses of primary lesions as evaluated according to the Japanese criteria were related to survival time, as were the responses of MML as evaluated according to the RECIST. Using the RECIST to evaluate treatment response may result in decreased numbers of assessable patients by excluding those with linitis plastica or undifferentiated types of gastric cancer, thereby biasing the demographic characteristics of subjects in clinical trials.
However, the subjects of our study received various kinds of chemotherapy over a very long period, and the data were collected retrospectively. This study thus did not have sufficient power to draw definitive conclusions. Our results must be validated prospectively in the future.

Acknowledgments This study did not receive any grants. We express our thanks to Mr. Peter Star of Medical Network K.K. for reviewing the English in this manuscript.

\section{References}

1. Therasse P, Arbuck SG, Eisenhauer EA, Wanders J, Kaplan RS, Rubinstein L, et al. New guidelines to evaluate the response to treatment in solid tumors. European Organization for Research and Treatment of Cancer, National Cancer Institute of the United States, National Cancer Institute of Canada. J Natl Cancer Inst 2000;92:205-16.

2. Japanese Gastric Cancer Association. Japanese classification of gastric carcinoma - 2nd English edition - Gastric Cancer 1998;1:10-24.

3. Koizumi W, Kurihara M, Tanabe S, Kondo I, Yamazaki I, Nonaka $\mathrm{M}$, et al. Advantages of Japanese response criteria for estimating the survival of patients with primary gastric cancer. Gastric Cancer 1999;2:14-9.

4. Kiyohashi A, Kurihara M, Yoshida S, Ohkubo T, Suga S. Antitumor effect and survival benefit of chemotherapy for unresectable advanced gastric cancer. Jpn J Clin Oncol 1993;23: 41-5.

5. Yoshida S, Miyata Y, Ohtsu A, Boku N, Shirao K, Shimada Y. Significance of and problems in adopting response evaluation criteria in solid tumor RECIST for assessing anticancer effects of advanced gastric cancer. Gastric Cancer 2000;3:128-33.

6. Ohtsu A, Boku N, Yoshida S, Miyata Y, Shirao K, Shimada Y, et al. Response of the primary lesion in gastric cancer to chemotherapeutic trials. Int J Clin Oncol 1998;3:3-6.

7. Anderson JR, Cain KC, Gelber RD. Analysis of survival by tumor response. J Clin Oncol 1983;11:710-9.

8. Maruyama K, Okabayashi K, Kinoshita T. Progress in gastric cancer surgery in Japan and its limits of radicality. World J Surg 1987;11:418-25.

9. Konishi T, Hiraishi M, Mafune K, Miyama T, Hirata T, Mori K, et al. Therapeutic efficacy and toxicity of sequential methotrexate and 5-fluorouracil in gastric cancer. Anticancer Res 1994;14: 1277-9.

10. Kikuchi S, Kida M, Kobayashi K, Yano T, Sakuramoto S, Watanabe $\mathrm{M}$, et al. New diagnostic imaging of gastrointestinal tumors: a preliminary study of three-dimensional tumor structure and volumetry. Anticancer Res 2005;25:2935-41.

11. Kim JH, Park SH, Hong HS, Auh YH. CT gastrography. Abdom Imaging 2005;30:509-17.

12. Kim AY, Kim HJ, Ha HK. Gastric cancer by multidetector row CT: preoperative staging. Abdom Imaging 2005;30:465-72. 